\title{
LEFT-INVARIANT MINIMAL UNIT VECTOR FIELDS ON THE SEMI-DIRECT PRODUCT $\mathbb{R}^{n} \rtimes_{P} \mathbb{R}$
}

\author{
SEUNGHUN Yi
}

\begin{abstract}
We provide the set of left-invariant minimal unit vector fields on the semi-direct product $\mathbb{R}^{n} \rtimes_{P} \mathbb{R}$, where $P$ is a nonsingular diagonal matrix and on the 7 classes of 4 -dimensional solvable Lie groups of the form $\mathbb{R}^{3} \rtimes_{P} \mathbb{R}$ which are unimodular and of type $(R)$.
\end{abstract}

\section{Introduction}

Let $(M, g)$ be a closed oriented Riemannian manifold and $\chi^{1}(M)$ be the set of all unit vector fields on $M$ which is assumed to be non-empty. Then an element $V \in \chi^{1}(M)$ can be viewed as a submanifold of the unit tangent sphere bundle $T^{1}(M)$ equipped with the Sasaki metric $g_{s}$ and the volume of the unit vector field is defined as the volume of this submanifold ([5]).

It is natural to try to find the unit vector fields of minimum volume among all unit vector fields on $M$. On the odd dimensional spheres, Gluck and Ziller ([5]) showed that the unit vector fields of minimum volume on $S^{3}$ are precisely the Hopf vector fields and no others ([5]). But on the higher dimensional spheres, $S^{2 n+1}, n \geq 2$, this is not the case ([4], [9], [13]).

The problem of finding unit vector fields which realize the minimum volume turns out to be very difficult, even for the spheres $S^{2 n+1}$. It leads to the consideration of the critical points of the volume functional restricted to $\chi^{1}(M)$.

Gil-Medriano and Llinares-Fuster proved that a unit vector field is a critical point of the volume functional if and only if the corresponding immersion in $\left(T^{1} M, g_{s}\right)$ is minimal ([3]). So we call such unit vector fields minimal even though the manifold is not compact.

A lot of examples of Riemannian manifolds and Lie groups equipped with minimal unit vector fields are provided ([1], [2], [3], [6], [7], [8], [15], [16], [17]). For the three dimensional Lie groups, Tsukada and Vanhecke provided the set of left-invariant minimal unit vector fields ([16]). But in most examples, the

Received March 10, 2009; Revised April 27, 2009.

2000 Mathematics Subject Classification. 53C20, 53C25, 53C42.

Key words and phrases. left-invariant minimal unit vector field, Lie group, semi-direct product. 
set of left-invariant minimal unit vector fields on Lie groups are not given, just some special left-invariant unit vector fields are shown to be minimal.

The aim of this paper is to provide the set of left-invariant minimal unit vector fields on some Lie groups. More precisely, we find the set of all leftinvariant minimal unit vector fields on the semi-direct product $\mathbb{R}^{n} \rtimes_{P} \mathbb{R}$ and on the 7 classes of 4-dimensional solvable Lie groups of the form $\mathbb{R}^{3} \rtimes_{P} \mathbb{R}$ which are unimodular and of type $(R)$.

The following is the main result.

Theorem 1.1. Let $P$ be a non-singular diagonal matrix $\operatorname{diag}\left\{p_{1}, p_{2}, \ldots, p_{n}\right\}$. Then the set of left-invariant minimal unit vector fields on the semi-direct product $\mathbb{R}^{n} \rtimes_{P} \mathbb{R}$ is

$$
\left\{E_{n+1}\right\} \cup\left(\mathcal{S} \cap\left\{E_{\alpha} \mid \alpha \in A_{1}\right\}_{\mathbb{R}}\right) \cup \cdots \cup\left(\mathcal{S} \cap\left\{E_{\alpha} \mid \alpha \in A_{k}\right\}_{\mathbb{R}}\right),
$$

where $\mathcal{S}$ is the unit sphere of the Lie algebra of the Lie group $\mathbb{R}^{n} \rtimes_{P} \mathbb{R}$.

The definitions of the sets $A_{1}, A_{2}, \ldots, A_{k}$ and $\left\{E_{\alpha} \mid \alpha \in A_{i}\right\}_{\mathbb{R}}$ in the above theorem will be given in Section 3 .

In Section 2, we give some basic notions and facts about minimal unit vector fields. In Section 3, we prove the main theorem. In Section 4, we provide the set of all left-invariant minimal unit vector fields on the Lie groups associated with the 4-dimensional solvable unimodular type $(R)$ Lie algebra of the form $\mathbb{R}^{3} \oplus_{P} \mathbb{R}$.

The author would like to express deep gratitude to the anonymous referee for valuable comments.

\section{Left-invariant minimal unit vector fields on a Lie group}

Let $(M, g)$ be a smooth Riemannian manifold, $\nabla$ be the Levi-Civita connection on $(M, g)$ and $R$ be the associated Riemannian curvature tensor with the sign convention $R_{X Y}=\nabla_{[X, Y]}-\left[\nabla_{X}, \nabla_{Y}\right]$.

We assume that the set $\chi^{1}(M)$ of unit vector fields on $M$ is non-empty. For $V \in \chi^{1}(M)$ we define the positive definite symmetric tensor field $L_{V}$ by

$$
L_{V}:=I+(\nabla V)^{*} \nabla V,
$$

where $I$ is the identity map and $(\nabla V)^{*}$ is the adjoint. Put $f(V)=\left(\operatorname{det} L_{V}\right)^{\frac{1}{2}}$. Then the volume functional $F: \chi^{1}(M) \longrightarrow \mathbb{R}$ is defined by

$$
F(V):=\int_{M} f(V) d v
$$

where $d v$ is the volume form on $(M, g)$.

Define a $(1,1)$-tensor field $K_{V}$ and a 1-form $\omega_{V}$ associated to $V$ as follows:

$$
\begin{aligned}
K_{V} & =f(V) \cdot L_{V}^{-1} \circ(\nabla V)^{*}, \\
\omega_{V}(X) & =\operatorname{tr}\left(Z \longmapsto \nabla_{Z} K_{V}\right)(X) .
\end{aligned}
$$


Definition 2.1. A unit vector field $\mathrm{V}$ on a Riemannian manifold $(M, g)$ is called minimal if $\omega_{V}(X)=0$ for all $X \in \mathcal{H}^{V}$.

Remark 2.1. In [3] it is proved that a unit vector field $V$ is a critical point for the volume functional $F$ if and only if the 1-form $\omega_{V}$ annihilates the distribution $\mathcal{H}^{V}$ consisting of tangent vectors orthogonal to $V$.

From now on we consider left-invariant unit vector fields on a Lie group $G$ equipped with a left-invariant metric. Then by the invariance with respect to the left translation, the function $f$ can be viewed as a function on the unit sphere $\mathcal{S}$ of the Lie algebra $\mathfrak{g}$. The distribution $\mathcal{H}^{V}$ can be identified with the orthogonal complement $V^{\perp}$ of $V$ in $\mathfrak{g}$ so $V^{\perp}$ is naturally identified with the tangent space $T_{V} \mathcal{S}$ of the unit sphere $\mathcal{S}$ at $V$. Thus a left-invariant unit vector field $V$ is minimal if and only if the 1-form $\omega_{V}$ on $\mathfrak{g}$ vanishes on $V^{\perp} \cong T_{V} \mathcal{S}$ ([16]). In [16] it is shown that

$$
\omega_{V}(X)=-d f_{V}(X)-\operatorname{tr} a d_{K_{V} X} .
$$

So $V$ is minimal if and only if $d f_{V}(X)=-\operatorname{tr} a d_{K_{V} X}$ for all $X \in T_{V} \mathcal{S}$. Thus on a unimodular Lie group $G$, i.e., $\operatorname{tr} a d_{X}=0$ for all $X \in \mathfrak{g}$, a left-invariant unit vector field $V$ is minimal if and only if $V$ is a critical point of the function $f$ on $\mathcal{S}$.

For a non-unimodular Lie group $G$, we consider its unimodular kernel $\mathcal{U}$ defined by

$$
\mathcal{U}=\left\{X \in \mathfrak{g} \mid \operatorname{tr} a d_{X}=0\right\} .
$$

Then $\mathcal{U}$ is an ideal of codimension 1 since $\operatorname{tr} a d_{X}$ is a linear functional. For a unit vector $H$ orthogonal to $\mathcal{U}$, the linear transformation $a d_{H}$ restricted to $\mathcal{U}$ is a derivation of $\mathcal{U}$. We have the following.

Proposition 2.2 ([16, Proposition 2.5]). Let $\mathcal{U}$ be the unimodular kernel of a non-unimodular Lie group such that $\left.a d_{H}\right|_{\mathcal{U}}$ is a symmetric endomorphism of $\mathcal{U}$ with respect to $\langle$,$\rangle . Then a left-invariant unit vector field V$ is minimal if and only if it is a critical point of the function $f$ on $\mathcal{S}$.

\section{The semi-direct product $\mathbb{R}^{n} \rtimes_{P} \mathbb{R}$}

Let $P=\left(p_{i j}\right) \in \mathrm{GL}(n, \mathbb{R})$ be a real $(n \times n)$-matrix. A homomorphism $\varphi: \mathbb{R} \rightarrow \operatorname{End}\left(\mathbb{R}^{n}\right)$ is defined by

$$
\varphi(\alpha)(x)=\alpha P x
$$

for $\alpha \in \mathbb{R}$ and $x \in \mathbb{R}^{n}$.

One can form a semi-direct product of the Lie algebra $\mathbb{R}$ by $\mathbb{R}^{n}$ as follows: The underlying linear space is the direct sum $\mathbb{R}^{n} \oplus \mathbb{R}$, and the bracket operation is given by

$$
[(a, \alpha),(b, \beta)]=(\varphi(\alpha) b-\varphi(\beta) a,[\alpha, \beta])=(\varphi(\alpha) b-\varphi(\beta) a, 0) .
$$


It is trivial to see that this does satisfy the skew-symmetry and the Jacobi identity. Denote the Lie algebra by $\mathbb{R}^{n} \oplus_{P} \mathbb{R}$ and the associated simply connected Lie group by $\mathbb{R}^{n} \rtimes_{P} \mathbb{R}$.

Put $E_{i}=(0, \ldots, 1, \ldots, 0) \in \mathbb{R}^{n+1}$. Let $\left\{E_{1}, \ldots, E_{n+1}\right\}$ be an orthonormal basis for $\mathfrak{g}=\mathbb{R}^{n} \oplus_{P} \mathbb{R}$ and equip the left-invariant metric on the associated Lie group.

For $X=\sum_{i=1}^{n+1} a_{i} E_{i}$, we have

$$
\operatorname{tr} a d_{X}=\sum_{j=1}^{n+1}\left\langle a d_{X} E_{j}, E_{j}\right\rangle=(\operatorname{tr} P) a_{n+1} .
$$

So $\mathbb{R}^{n} \rtimes_{P} \mathbb{R}$ is unimodular if and only if $\operatorname{tr} P=0$. If $\operatorname{tr} P \neq 0$, then the unimodular kernel is $\mathcal{U}=\left\{X \in \mathfrak{g} \mid X=\sum_{i=1}^{n} a_{i} E_{i}\right\}$ and a unit vector field orthogonal to $\mathcal{U}$ is $E_{n+1}$.

For an element $X \in \mathcal{U}$ we have $a d_{H}(X)=X$ and thus $\left.a d_{H}\right|_{\mathcal{U}}=\left.I d\right|_{\mathcal{U}}$. Therefore $\left.a d_{H}\right|_{\mathcal{U}}$ is a symmetric endomorphism of $\mathcal{U}$ with respect to $\langle$,$\rangle . So by$ the Proposition 2.2, a left-invariant unit vector field $V$ is minimal if and only if $V$ is a critical point of $f$ on $\mathcal{S}$.

For a matrix $P=\left(p_{i j}\right)$ we have the following.

Proposition 3.1. For $1 \leq i, j \leq n$, we have

(i) $\nabla_{E_{i}} E_{j}=\frac{1}{2}\left(p_{i j}+p_{j i}\right) E_{n+1}$;

(ii) $\nabla_{E_{i}} E_{n+1}=-\frac{1}{2} \sum_{k=1}^{n}\left(p_{k i}+p_{i k}\right) E_{k}$;

(iii) $\nabla_{E_{n+1}} E_{i}=\frac{1}{2} \sum_{k=1}^{n}\left(p_{k i}-p_{i k}\right) E_{k}$;

(iv) $\nabla_{E_{n+1}} E_{n+1}=0$.

Thus we have

$$
\begin{aligned}
\nabla E_{i} & =\frac{1}{2} \sum_{j=1}^{n}\left(p_{i j}+p_{j i}\right) E_{n+1} \otimes \theta_{j}+\frac{1}{2} \sum_{j=1}^{n}\left(p_{j i}-p_{i j}\right) E_{j} \otimes \theta_{n+1}, \\
\nabla E_{n+1} & =-\frac{1}{2} \sum_{j=1}^{n}\left(\sum_{i=1}^{n}\left(p_{i j}+p_{j i}\right) E_{i}\right) \otimes \theta_{j} .
\end{aligned}
$$

Proof of Theorem 1.1. Assume that the matrix $P$ is a non-singular diagonal matrix $\operatorname{diag}\left\{p_{1}, p_{2}, \ldots, p_{n}\right\}$. Then for $V=\sum_{i=1}^{n+1} a_{i} E_{i}$ we have

$$
\nabla V=\left(\begin{array}{ccccc}
-p_{1} a_{n+1} & 0 & \cdots & 0 & 0 \\
0 & -p_{2} a_{n+1} & \cdots & 0 & 0 \\
\vdots & \vdots & \ddots & \vdots & \vdots \\
0 & 0 & \cdots & -p_{n} a_{n+1} & 0 \\
p_{1} a_{1} & p_{2} a_{2} & \cdots & p_{n} a_{n} & 0
\end{array}\right) .
$$

So we have

$\operatorname{det}\left(I+(\nabla V)^{*} \nabla V\right)$ 


$$
=\left(\begin{array}{ccccc}
1+\left(a_{1}^{2}+a_{n+1}^{2}\right) p_{1}^{2} & a_{1} a_{2} p_{1} p_{2} & \cdots & a_{1} a_{n} p_{1} p_{n} & 0 \\
a_{1} a_{2} p_{1} p_{2} & 1+\left(a_{2}^{2}+a_{n+1}^{2}\right) p_{2}^{2} & \cdots & a_{2} a_{n} p_{2} p_{n} & 0 \\
\vdots & \vdots & \ddots & \vdots & \vdots \\
a_{1} a_{n} p_{1} p_{n} & a_{2} a_{n} p_{2} p_{n} & \cdots & 1+\left(a_{n}^{2}+a_{n+1}^{2}\right) p_{n}^{2} & 0 \\
0 & 0 & \cdots & 0 & 1
\end{array}\right) .
$$

Put $H\left(a_{1}, a_{2}, \ldots, a_{n+1}\right):=\operatorname{det}\left(I+(\nabla V)^{*} \nabla V\right)$. Then we have the following.

\section{Lemma 3.2.}

$$
\begin{aligned}
& H\left(a_{1}, a_{2}, \ldots, a_{n+1}\right) \\
= & \left(1+a_{n+1}^{2} p_{1}^{2}\right) \cdots\left(1+a_{n+1}^{2} p_{n}^{2}\right)+\sum_{i=1}^{n} a_{i}^{2} p_{i}^{2} \cdot \frac{\Pi_{j=1}^{n}\left(1+a_{n+1}^{2} p_{j}^{2}\right)}{1+a_{n+1}^{2} p_{i}^{2}} .
\end{aligned}
$$

Proof. By the properties of determinant we have

$$
\begin{aligned}
& H\left(a_{1}, a_{2}, \ldots, a_{n+1}\right) \\
& =\operatorname{det}\left(\begin{array}{cccc}
1+a_{n+1}^{2} p_{1}^{2} & 0 & \cdots & 0 \\
a_{1} a_{2} p_{1} p_{2} & 1+\left(a_{2}^{2}+a_{n+1}^{2}\right) p_{2}^{2} & \cdots & a_{2} a_{n} p_{2} p_{n} \\
\vdots & \vdots & \ddots & \vdots \\
a_{1} a_{n} p_{1} p_{n} & a_{2} a_{n} p_{2} p_{n} & \cdots & 1+\left(a_{n}^{2}+a_{n+1}^{2}\right) p_{n}^{2}
\end{array}\right) \\
& +\operatorname{det}\left(\begin{array}{cccc}
a_{1}^{2} p_{1}^{2} & a_{1} a_{2} p_{1} p_{2} & \cdots & a_{1} a_{n} p_{1} p_{n} \\
a_{1} a_{2} p_{1} p_{2} & 1+\left(a_{2}^{2}+a_{n+1}^{2}\right) p_{2}^{2} & \cdots & a_{2} a_{n} p_{2} p_{n} \\
\vdots & \vdots & \ddots & \vdots \\
a_{1} a_{n} p_{1} p_{n} & a_{2} a_{n} p_{2} p_{n} & \cdots & 1+\left(a_{n}^{2}+a_{n+1}^{2}\right) p_{n}^{2}
\end{array}\right) \\
& =\left(1+a_{n+1}^{2} p_{1}^{2}\right) H\left(a_{2}, a_{3}, \ldots, a_{n+1}\right) \\
& +a_{1}^{2} p_{1}^{2} \operatorname{det}\left(\begin{array}{ccccc}
1 & a_{2} p_{2} & a_{3} p_{3} & \cdots & a_{n} p_{n} \\
0 & 1+a_{n+1}^{2} p_{2}^{2} & 0 & \cdots & 0 \\
0 & 0 & 1+a_{n+1}^{2} p_{3}^{2} & \cdots & 0 \\
\vdots & \vdots & \vdots & \ddots & \vdots \\
0 & 0 & 0 & \cdots & 1+a_{n+1}^{2} p_{n}^{2}
\end{array}\right) \\
& =\left(1+a_{n+1}^{2} p_{1}^{2}\right) H\left(a_{2}, \ldots, a_{n+1}\right)+a_{1}^{2} p_{1}^{2}\left(1+a_{n+1}^{2} p_{2}^{2}\right) \cdots\left(1+a_{n+1}^{2} p_{n}^{2}\right) \\
& =\left(1+a_{n+1}^{2} p_{1}^{2}\right)\left[\left(1+a_{n+1}^{2} p_{2}^{2}\right) H\left(a_{3}, \ldots, a_{n+1}\right)\right. \\
& \left.+a_{2}^{2} p_{2}^{2}\left(1+a_{n+1}^{2} p_{3}^{2}\right) \cdots\left(1+a_{n+1}^{2} p_{n}^{2}\right)\right] \\
& +a_{1}^{2} p_{1}^{2}\left(1+a_{n+1}^{2} p_{2}^{2}\right) \cdots\left(1+a_{n+1}^{2} p_{n}^{2}\right) \\
& =\left(1+a_{n+1}^{2} p_{1}^{2}\right) \cdots\left(1+a_{n+1}^{2} p_{n-2}^{2}\right) H\left(a_{n-1}, a_{n}, a_{n+1}\right)+\sum_{i=1}^{n-2} a_{i}^{2} p_{i}^{2} \cdot \frac{\Pi_{j=1}^{n}\left(1+a_{n+1}^{2} p_{j}^{2}\right)}{1+a_{n+1}^{2} p_{i}^{2}} \\
& =\left(1+a_{n+1}^{2} p_{1}^{2}\right) \cdots\left(1+a_{n+1}^{2} p_{n}^{2}\right)+\sum_{i=1}^{n} a_{i}^{2} p_{i}^{2} \cdot \frac{\Pi_{j=1}^{n}\left(1+a_{n+1}^{2} p_{j}^{2}\right)}{1+a_{n+1}^{2} p_{i}^{2}} .
\end{aligned}
$$


In the last equality of the above equations, we used the following identity.

$H\left(a_{n-1}, a_{n}, a_{n+1}\right)=a_{n-1}^{2} p_{n-1}^{2}\left(1+a_{n+1}^{2} p_{n}^{2}\right)+\left(1+a_{n+1}^{2} p_{n-1}^{2}\right)\left(1+\left(a_{n}^{2}+a_{n+1}^{2}\right) p_{n}^{2}\right)$.

Moreover, for $1 \leq i \leq n$, we have

$$
\frac{\partial}{\partial a_{i}} H\left(a_{1}, \ldots, a_{n+1}\right)=2 a_{i} p_{i}^{2} \cdot \frac{\Pi_{j=1}^{n}\left(1+a_{n+1}^{2} p_{j}^{2}\right)}{1+a_{n+1}^{2} p_{i}^{2}} .
$$

Since the sets of critical points of the functionals $H$ and $F=\sqrt{H}$ are equal, we will find the set of critical points of the function $H\left(a_{1}, \ldots, a_{n+1}\right)$ with constraint $g\left(a_{1}, a_{2}, \ldots, a_{n+1}\right):=a_{1}^{2}+a_{2}^{2}+\cdots+a_{n+1}^{2}-1=0$ by using the Lagrangian multiplier method. Thus we have to solve the following simultaneous equation.

$$
\begin{cases}\nabla H & =\lambda \nabla g \\ g & =0\end{cases}
$$

More precisely,

$$
\begin{aligned}
2 a_{i} p_{i}^{2} \cdot \frac{\Pi_{j=1}^{n}\left(1+a_{n+1}^{2} p_{j}^{2}\right)}{1+a_{n+1}^{2} p_{i}^{2}} & =2 \lambda a_{i}, 1 \leq i \leq n, \\
\frac{\partial H}{\partial a_{n+1}} & =2 \lambda a_{n+1}, \\
a_{1}^{2}+a_{2}^{2}+\cdots+a_{n+1}^{2}-1 & =0 .
\end{aligned}
$$

Since $P$ is non-singular, we have $p_{i} \neq 0,1 \leq i \leq n$.

Claim 3.3. $\lambda \neq 0$.

Proof. If $\lambda=0$, then by the equation (3-1) we have $a_{i}=0,1 \leq i \leq n$. By the Lemma 3.2 we have

$$
H\left(0,0, \ldots, 0, a_{n+1}\right)=\left(1+a_{n+1}^{2} p_{1}^{2}\right)\left(1+a_{n+1}^{2} p_{2}^{2}\right) \cdots\left(1+a_{n+1}^{2} p_{n}^{2}\right) .
$$

Thus we have

$$
\begin{aligned}
& \frac{\partial H}{\partial a_{n+1}}\left(0,0, \ldots, 0, a_{n+1}\right) \\
& =2 a_{n+1} \times\left\{p_{1}^{2}\left(1+\widehat{a_{n+1}^{2} p_{1}^{2}}\right)\left(1+a_{n+1}^{2} p_{2}^{2}\right) \cdots\left(1+a_{n+1}^{2} p_{n}^{2}\right)\right. \\
& +p_{2}^{2}\left(1+a_{n+1}^{2} p_{1}^{2}\right)\left(1+\widehat{a_{n+1}^{2} p_{2}^{2}}\right) \cdots\left(1+a_{n+1}^{2} p_{n}^{2}\right) \\
& \left.+\cdots+p_{n}^{2}\left(1+a_{n+1}^{2} p_{1}^{2}\right)\left(1+a_{n+1}^{2} p_{2}^{2}\right) \cdots\left(1+\widehat{a_{n+1}^{2} p_{n}^{2}}\right)\right\},
\end{aligned}
$$

where the symbol ${ }^{\wedge}$ over $a_{n+1}^{2} p_{i}^{2}$ indicates that it is omitted.

Thus by the equation $(3-2)$ we have $a_{n+1}=0$. This contradicts to the equation (3-3). Thus we have $\lambda \neq 0$.

Now consider the case $a_{n+1} \neq 0$.

Assume that $a_{i} \neq 0,1 \leq i \leq n$. Then by the equation (3-1) we have

$$
\lambda=p_{i}^{2}\left(1+a_{n+1}^{2} p_{1}^{2}\right) \cdots\left(1+\widehat{a_{n+1}^{2} p_{i}^{2}}\right) \cdots\left(1+a_{n+1}^{2} p_{n}^{2}\right) .
$$


But by Lemma 3.2, we have

$$
\begin{aligned}
\frac{\partial H}{\partial a_{n+1}}= & \sum_{i=1}^{n} 2 a_{n+1} p_{i}^{2} \cdot \frac{\Pi_{j=1}^{n}\left(1+a_{n+1}^{2} p_{j}^{2}\right)}{1+a_{n+1}^{2} p_{i}^{2}} \\
& +\sum_{i=1}^{n} a_{i}^{2} p_{i}^{2} \cdot\left[\sum_{1 \leq j \leq n, j \neq i} 2 a_{n+1} p_{j}^{2} \cdot \frac{\Pi_{j=1}^{n}\left(1+a_{n+1}^{2} p_{j}^{2}\right)}{\left(1+a_{n+1}^{2} p_{i}^{2}\right)\left(1+a_{n+1}^{2} p_{j}^{2}\right)}\right] .
\end{aligned}
$$

So by the equation (3-2), we have

$$
\begin{aligned}
\lambda= & \sum_{i=1}^{n} p_{i}^{2} \cdot \frac{\Pi_{j=1}^{n}\left(1+a_{n+1}^{2} p_{j}^{2}\right)}{1+a_{n+1}^{2} p_{i}^{2}} \\
& +\sum_{i=1}^{n} a_{i}^{2} p_{i}^{2} \cdot\left[\sum_{1 \leq j \leq n, j \neq i} p_{j}^{2} \cdot \frac{\Pi_{j=1}^{n}\left(1+a_{n+1}^{2} p_{j}^{2}\right)}{\left(1+a_{n+1}^{2} p_{i}^{2}\right)\left(1+a_{n+1}^{2} p_{j}^{2}\right)}\right] .
\end{aligned}
$$

Comparing two equations (3-4) and (3-5) above, we get a contradiction. Thus we have $a_{i}=0, i=1,2, \ldots, n$. So we have $a_{n+1}= \pm 1$ and thus $\pm E_{n+1}$ are minimal unit vectors.

Let's consider the case $a_{n+1}=0$.

Partition the set $\{1,2, \ldots, n\}$ into relatively disjoint sets $A_{1}, A_{2}, \ldots, A_{k}$ which satisfy the following properties.

(i) For $\alpha \in A_{i}, \beta \in A_{j}, i \neq j$, we have $p_{\alpha}^{2} \neq p_{\beta}^{2}$.

(ii) For $\alpha_{1}, \alpha_{2} \in A_{i}$, we have $p_{\alpha_{1}}^{2}=p_{\alpha_{2}}^{2}$.

Now let $\alpha_{i} \in A_{i}, 1 \leq i \leq k$, and consider the following set of reduced simultaneous equation

$$
2 a_{\alpha_{i}} p_{\alpha_{i}}^{2}=2 \lambda a_{\alpha_{i}}, 1 \leq i \leq k .
$$

For $\alpha \in A_{i}, \beta \in A_{j}, i \neq j$, we have $p_{\alpha}^{2} \neq p_{\beta}^{2}$. So if $a_{\beta} \neq 0$ then $a_{\alpha}=0$ for all $i \neq j$.

Thus the set of minimal unit vector fields are as follows.

$$
\left(\mathcal{S} \cap\left\{E_{\alpha} \mid \alpha \in A_{1}\right\}_{\mathbb{R}}\right) \cup \cdots \cup\left(\mathcal{S} \cap\left\{E_{\beta} \mid \beta \in A_{k}\right\}_{\mathbb{R}}\right),
$$

where $\mathcal{S}$ is the unit sphere of the Lie algebra of the Lie group $\mathbb{R}^{n} \rtimes_{P} \mathbb{R}$ and $\left\{E_{\alpha} \mid \alpha \in A_{i}\right\}_{\mathbb{R}}$ is the subspace spanned by $\left\{E_{\alpha} \mid \alpha \in A_{i}\right\}$. This completes the proof.

Corollary 3.4. Let $P$ be a diagonal matrix $\operatorname{diag}\left\{p_{1}, p_{2}, \ldots, p_{n}\right\}$ such that all $p_{i}$ 's are non-zero and relatively distinct. Then the set of left-invariant minimal unit vector fields on the semi-direct product $\mathbb{R}^{n} \rtimes_{P} \mathbb{R}$ is as follows:

$$
\left\{ \pm E_{1}, \pm E_{2}, \ldots, \pm E_{n+1}\right\} \text {. }
$$


Corollary 3.5. Let $P$ be a matrix of the form $c I_{n}$, where $c$ is a nonzero constant and $I_{n}$ is the $n \times n$ identity matrix. Then the set of left-invariant minimal unit vector fields on the semi-direct product $\mathbb{R}^{n} \rtimes_{P} \mathbb{R}$ is

$$
\left(\mathcal{S} \cap E_{n+1}^{\perp}\right) \cup\left\{ \pm E_{n+1}\right\} .
$$

\section{The 4-dimensional solvable unimodular type $(R)$ Lie groups}

A connected Lie group $G$ is of type $(R)$ if $\operatorname{ad}(X): \mathfrak{g} \rightarrow \mathfrak{g}$ has only real eigenvalues for every $X \in \mathfrak{g}$. And it is unimodular if $\operatorname{ad}(X)$ has trace 0 for every $X \in \mathfrak{g}$. For $\mathbb{R}^{3} \rtimes_{P} \mathbb{R}$, there are 7 classes of solvable unimodular Lie groups of type $(R)$, up to conjugation and scalar multiple. The following table is the list of 7 isomorphism classes of 4-dimensional solvable unimodular type $(R)$ Lie algebras of the form $\mathbb{R}^{3} \oplus_{P} \mathbb{R}$ and the associated simply connected Lie groups $\mathbb{R}^{3} \rtimes_{P} \mathbb{R}([11])$ :

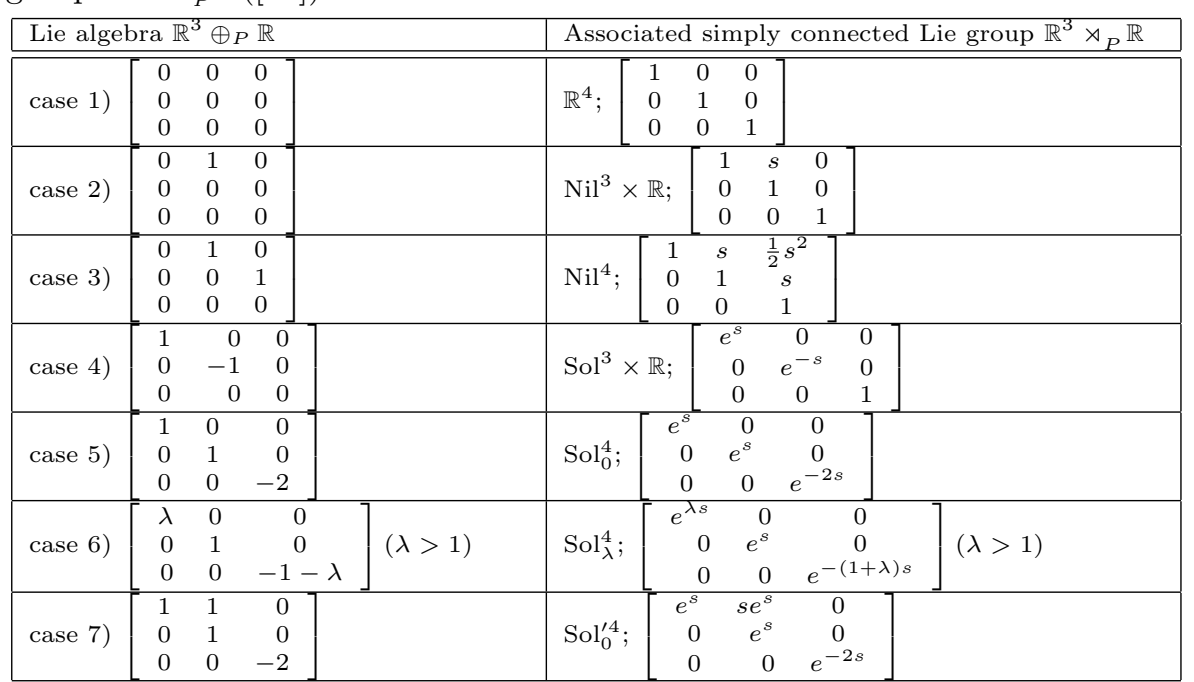

It is straightforward to determine the left-invariant minimal unit vector fields on the Lie groups above. But as an example we show the calculations for the case 4 .

For $V=\sum_{i=1}^{4} a_{i} E_{i}, a_{1}^{2}+a_{2}^{2}+a_{3}^{2}+a_{4}^{2}=1$, we have

$$
\nabla V=\left(\begin{array}{cccc}
-a_{4} & 0 & 0 & 0 \\
0 & a_{4} & 0 & 0 \\
0 & 0 & 0 & 0 \\
a_{1} & -a_{2} & 0 & 0
\end{array}\right)
$$

and

$$
L_{V}:=I+(\nabla V)^{*} \nabla V=\left(\begin{array}{cccc}
1+a_{1}^{2}+a_{4}^{2} & -a_{1} a_{2} & 0 & 0 \\
-a_{1} a_{2} & 1+a_{2}^{2}+a_{4}^{2} & 0 & 0 \\
0 & 0 & 1 & 0 \\
0 & 0 & 0 & 1
\end{array}\right)
$$


So we have

$$
f(V):=\left(\operatorname{det} L_{V}\right)^{1 / 2}=\sqrt{\left(1+a_{4}^{2}\right)\left(1+a_{1}^{2}+a_{2}^{2}+a_{4}^{2}\right)} .
$$

By the Lagrangian multiplier method, we have to solve the following simultaneous equation.

$$
\begin{aligned}
& \frac{a_{1}\left(1+a_{4}^{2}\right)}{\sqrt{\left(1+a_{4}^{2}\right)\left(1+a_{1}^{2}+a_{2}^{2}+a_{4}^{2}\right)}}=2 \lambda a_{1}, \\
& \frac{a_{2}\left(1+a_{4}^{2}\right)}{\sqrt{\left(1+a_{4}^{2}\right)\left(1+a_{1}^{2}+a_{2}^{2}+a_{4}^{2}\right)}}=2 \lambda a_{2}, \\
& 0=2 \lambda a_{3}, \\
& \frac{a_{4}\left(2+a_{1}^{2}+a_{2}^{2}+2 a_{4}^{2}\right)}{\sqrt{\left(1+a_{4}^{2}\right)\left(1+a_{1}^{2}+a_{2}^{2}+a_{4}^{2}\right)}}=2 \lambda a_{4} .
\end{aligned}
$$

If $\lambda=0$, then we have $a_{1}=a_{2}=a_{4}=0$ by the equations (4-1), (4-2), and (4-4). So we have $a_{3}= \pm 1$ and thus $\pm E_{3}$ is minimal.

Now assume that $\lambda \neq 0$. By the equation (4-3) we have $a_{3}=0$. If $a_{4} \neq 0$, then we have $a_{1}=a_{2}=0$ and thus $\pm E_{4}$ is minimal. If $a_{4}=0$, then we have $a_{1}^{2}+a_{2}^{2}=1$ and thus $\left\{a_{1} E_{1}+a_{2} E_{2} \mid a_{1}^{2}+a_{2}^{2}=1\right\}$ is the set of minimal unit vector fields.

Therefore the set of left-invariant minimal unit vector fields is

$$
\left\{a_{1} E_{1}+a_{2} E_{2} \mid a_{1}^{2}+a_{2}^{2}=1\right\} \cup\left\{ \pm E_{3}\right\} \cup\left\{ \pm E_{4}\right\} .
$$

Similarly we can determine the set of left-invariant minimal unit vector fields in the other cases.

Theorem 4.1. The sets of left-invariant minimal unit vector fields on the Lie groups associated with the 4-dimensional solvable unimodular type $(R)$ Lie algebra of the form $\mathbb{R}^{n} \rtimes_{P} \mathbb{R}$ are as follows:

Case 1) Every left-invariant unit vector fields are minimal;

Case 2) $\left\{a_{1} E_{1}+a_{2} E_{2}+a_{4} E_{4} \mid a_{1}^{2}+a_{2}^{2}+a_{4}^{2}=1\right\} \cup\left\{ \pm E_{3}\right\}$;

Case 3) $\left\{ \pm \frac{1}{2} \sqrt{2 \pm \sqrt{2}}\left((-1 \pm \sqrt{2}) E_{1}+E_{2}\right)\right\} \cup\left\{ \pm E_{3}\right\} \cup\left\{ \pm E_{4}\right\}$;

Case 4) $\left\{a_{1} E_{1}+a_{2} E_{2} \mid a_{1}^{2}+a_{2}^{2}=1\right\} \cup\left\{ \pm E_{3}\right\} \cup\left\{ \pm E_{4}\right\}$;

Case 5) $\left\{a_{1} E_{1}+a_{2} E_{2} \mid a_{1}^{2}+a_{2}^{2}=1\right\} \cup\left\{ \pm E_{3}\right\} \cup\left\{ \pm E_{4}\right\}$;

Case 6) $\left\{ \pm E_{1}, \pm E_{2}, \pm E_{3}, \pm E_{4}\right\}$;

Case 7) $\left\{ \pm \frac{1}{\sqrt{2}}\left(E_{1}+E_{2}\right)\right\} \cup\left\{ \pm \frac{1}{\sqrt{2}}\left(E_{1}-E_{2}\right)\right\} \cup\left\{ \pm E_{3}\right\} \cup\left\{ \pm E_{4}\right\}$. 


\section{References}

[1] E. Boeckx and L. Vanhecke, Harmonic and minimal radial vector fields, Acta Math. Hungar. 90 (2001), no. 4, 317-331.

[2] _ Harmonic and minimal vector fields on tangent and unit tangent bundles, Differential Geom. Appl. 13 (2000), no. 1, 77-93.

[3] O. Gil-Medriano and E. Llinares-Fuster, Minimal unit vector fields, Tohoku Math. J. (2) 54 (2002), no. 1, 71-84.

[4] - Second variation of volume and energy of vector fields. Stability of Hopf vector fields, Math. Ann. 320 (2001), no. 3, 531-545.

[5] H. Gluck and W. Ziller, On the volume of a unit vector field on the three-sphere, Comment. Math. Helv. 61 (1986), no. 2, 177-192.

[6] J. C. Gonzalez-Davila and L. Vanhecke, Examples of minimal unit vector fields, Special issue in memory of Alfred Gray (1939-1998), Ann. Global Anal. Geom. 18 (2000), no. 3-4, 385-404.

[7] _ Minimal and harmonic characteristic vector fields on three-dimensional contact metric manifolds, J. Geom. 72 (2001), no. 1-2, 65-76.

[8] _ Energy and volume of unit vector fields on three-dimensional Riemannian manifolds, Differential Geom. Appl. 16 (2002), no. 3, 225-244.

[9] D. L. Johnson, Volumes of flows, Proc. Amer. Math. Soc. 104 (1988), no. 3, 923-931.

[10] O. Kowalski and Z. Vlasek, omogeneous Riemannian manifolds with only one homogeneous geodesic, Publ. Math. Debrecen 62 (2003), no. 3-4, 437-446.

[11] J. B. Lee, K. B. Lee, J. Shin, and S. Yi, Unimodular groups of type $\mathbb{R}^{3} \rtimes \mathbb{R}$, J. Korean Math. Soc. 44 (2007), no. 5, 1121-1137.

[12] J. Milnor, Curvatures of left invariant metrics on Lie groups, Advances in Math. 21 (1976), no. 3, 293-329.

[13] S. L. Pedersen, Volumes of vector fields on spheres, Trans. Amer. Math. Soc. 336 (1993), no. $1,69-78$.

[14] W. A. Poor, Differential Geometric Structures, McGraw-Hill Book Co., New York, 1981.

[15] M. Salvai, OOn the volume of unit vector fields on a compact semisimple Lie group, J. Lie Theory 13 (2003), no. 2, 457-464.

[16] K. Tsukada and L. Vanhecke, Invariant minimal unit vector fields on Lie groups, Period. Math. Hungar. 40 (2000), no. 2, 123-133.

[17] S. Yi, Left-invariant minimal unit vector fields on a Lie group of constant negative sectional curvature, Bull. Korean Math. Soc. 46 (2009), no. 4, 713-720.

Department of Mathematics

SCIENCES AND LIBERAL ARTS

YOUNGDONG UNIVERSITY

YOUNGDONG 370-701, KorEA

E-mail address: seunghun@youngdong.ac.kr 